\title{
Breve reseña histórica de la microscopía electrónica en México y el mundo*
}

\section{Brief history of electron microscopy in Mexico and the world}

\author{
José Reyes Gasga**
}

\begin{abstract}
The contribution of the electron microscope to nanosciences has been exceptional since it is the type of equipment that allows the study and analysis of nanometric materials. A brief historical review of the discovery and evolution of the transmission electron microscope (TEM) and scanning electron microscope (SEM) and the installation in Mexico of these electron microscopes is presented. The writing is in such a way that the text and illustrations complement each other.
\end{abstract}

KEYWORDS: electron microscopy, transmission electron microscopy, scanning electron microscopy, electron microscopy in Mexico.

RESUMEN: La contribución del microscopio electrónico a las nanociencias ha sido excepcional por ser el equipo que permite estudiar y analizar materiales nanométricos. En este trabajo se presenta una breve reseña histórica sobre el descubrimiento y evolución del microscopio electrónico de transmisión (TEM, por sus siglas en inglés) y el electrónico de barrido (SEM, por sus siglas en inglés), así como la instalación de estos microscopios electrónicos en México. El escrito se presenta de tal manera que el texto y las ilustraciones se complementen.

PALABRAS CLAVE: microscopía electrónica, microscopio electrónico de transmisión, microscopio electrónico de barrido, reseña histórica, microscopía electrónica en México.

\section{Introducción}

Un concepto importante en el campo de la microscopía es la resolución, la cual se define como la distancia más pequeña que es posible observar con un instrumento óptico, y depende de varios factores como, la longitud de onda de la fuente de iluminación y los defectos de las lentes. Entre estos están las aberraciones esféricas, cromáticas y astigmatismo. A medida que se reducen la longitud de onda y los defectos de las lentes la resolución se mejora. Actualmente, la resolución del microscopio electrónico de barrido (SEM, por

* Algunas de las ilustraciones presentadas son del dominio público y fueron tomadas de www. google.com.mx/search

** Universidad Nacional Autónoma de México, Instituto de Física. Circuito de la Investigación s/n, Ciudad Universitaria, 04510 Coyoacán, Ciudad de México, México.

Correo electrónico: jreyes@fisica.unam.mx 
sus siglas en inglés) ha alcanzado $1 \mathrm{~nm}$, mientras que el microscopio electrónico de transmisión (TEM, por sus siglas en inglés) ha alcanzado valores menores a $0.1 \mathrm{~nm}$. Por lo tanto, el microscopio electrónico es el equipo indicado si queremos estudiar y analizar materiales nanométricos, su contribución en las nanociencias ha sido excepcional.

La observación detallada de objetos pequeños por debajo de la resolución del ojo humano (la cual es de aproximadamente $1 \mathrm{~mm}$ ) siempre ha estado entre los mayores desafíos del ser humano y, sin lugar a duda, el microscopio electrónico, tanto el TEM como el SEM, ha figurado entre sus principales herramientas (figura 1). "Hasta no ver, no creer", esta frase adjudicada a Santo Tomás (aunque bien la pudo haber dicho cualquier persona desde los comienzos de la historia) marca la importancia que damos a nuestra vista en el desarrollo del conocimiento. Esta frase ha marcado el rumbo seguido en la microscopía y, también debe ser dicho, en la astronomía.

El microscopio de luz fue inventado por el fabricante de lentes holandés Zacarias Janssen en 1595, aunque se discute su paternidad con el italiano Galileo Galilei. En 1665, Robert Hooke publicó el libro Micrographia, donde presentó sus "dibujos pequeños" vistos a través de un microscopio de luz. En 1742, el inglés John Cuff diseñó un microscopio compuesto, popularizado en el libro de Henry Baker The microscope made easy. Tomando en cuenta la resolución del microscopio de luz donde se usa luz con longitudes de onda de alrededor de 500 $\mathrm{nm}$, en 1897, Ernst Abbe (figura 2), uno de los pilares en el desarrollo de la microscopía de luz, indicó que se había alcanzado la máxima resolución posible del

Figura 1. Todos los microscopios tienen el mismo objetivo: ver el mundo microscópico y nanoscópico con el mayor detalle posible.

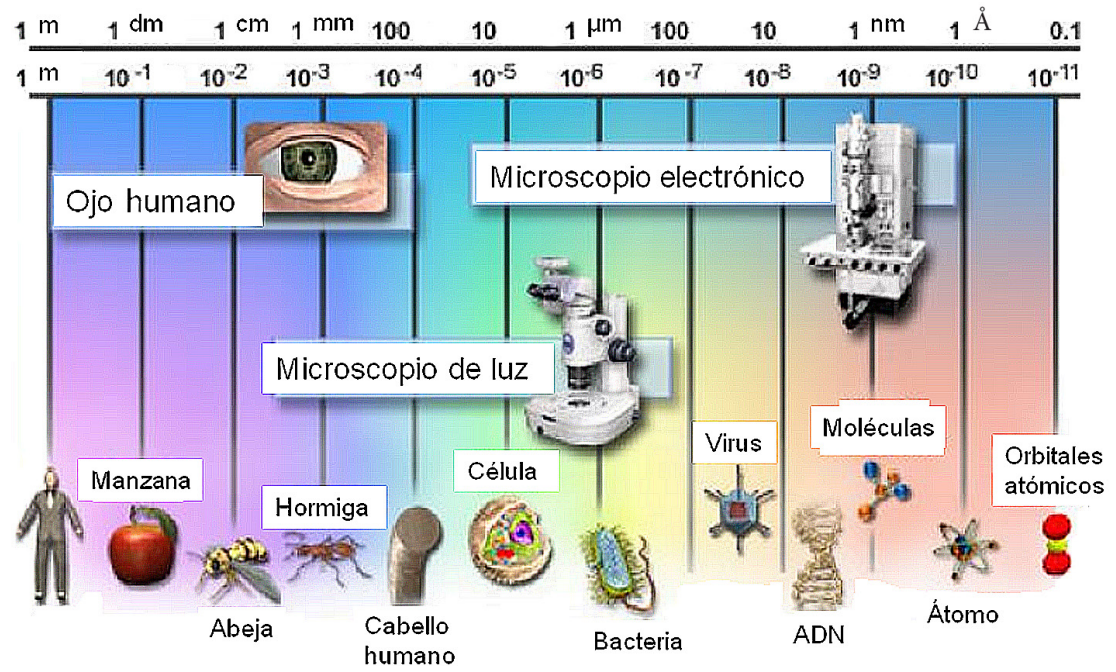

Fuente: www.biogeo.esy.es 
Figura 2. Ernst Abbe, padre de la microscopía de luz moderna. En 1897 Abbe comentó que "la microscopía de luz ha alcanzado el límite de resolución".

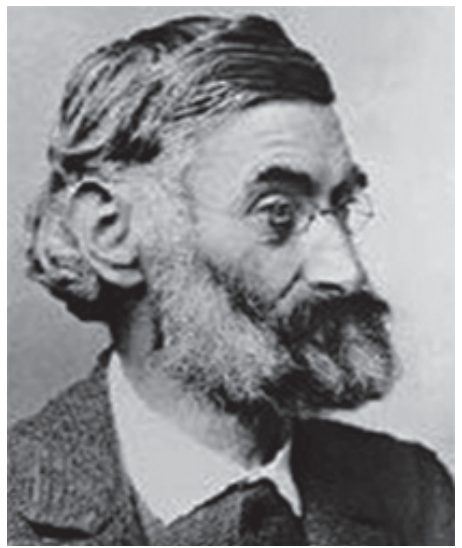

Fuente: www.google.com.mx/search

microscopio de luz (unas décimas de micra). Obtener imágenes por debajo de $200 \mathrm{~nm}$ de resolución solo sería posible en un microscopio que usara una iluminación con longitudes de onda más pequeñas que las de la luz (Brenton, 2004; Nixon, 1998).

En 1895, Wilhelm Conrad Röntgen descubre los rayos $\mathrm{X}$ y se inicia una vorágine de experimentos con el objetivo de determinar su origen y naturaleza. De esta manera, Joseph John Thomson descubrió los electrones (del griego elektron: ámbar) en 1897, al realizar estudios con tubos de rayos catódicos (figura 3). Los electrones son partículas cargadas negativamente (con carga de $-1.6 \mathrm{x}$ $10^{-19} \mathrm{C}$ y masa de $9.1 \times 10^{-31} \mathrm{Kg}$ ) las cuales giran alrededor del núcleo, y son estas partículas las que tendrían un papel decisivo en el desarrollo de un nuevo tipo de microscopios. En un principio solo se observaron sus manifestaciones corpusculares puesto que en ese momento no existía en el campo de la física la idea de que las partículas pudieran presentar un comportamiento ondulatorio. A principios de siglo XX, se desarrolla la mecánica cuántica cuando Max Planck descubre que la radiación electromagnética es emitida en paquetes de energía y Albert Einstein indica que la luz visible está constituida de cuantos de energía llamados fotones.

Figura 3. En 1897, el físico inglés Joseph John Thompson descubre el electrón.
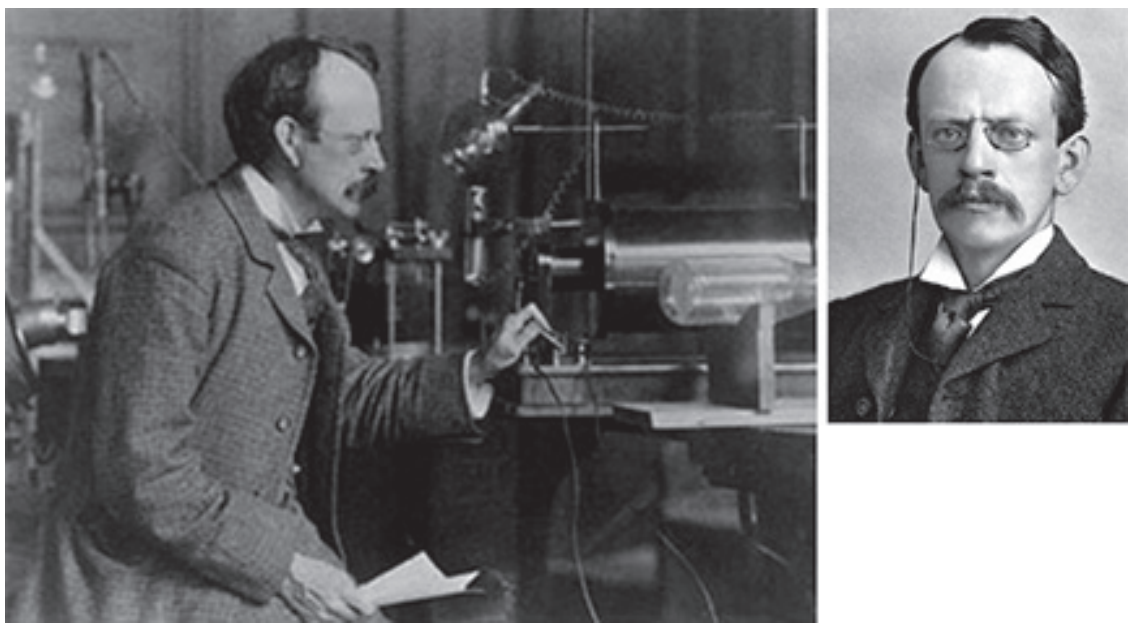

Fuente: www.google.com.mx/search 
En 1924, Luis de Broglie (figura 4) sacude los cimientos de la física de su época al enunciar el carácter ondulatorio de los electrones y asignarles longitudes de ondas de acuerdo con la velocidad que presentan (por ejemplo, en un microscopio electrónico de $100 \mathrm{kV}$, la longitud de onda de los electrones es de $0.0037 \mathrm{~nm}$ ). Este planteamiento teórico, que luego fue confirmado experimentalmente en 1926 por Davisson y Germer, sentó las bases para el desarrollo de la mecánica ondulatoria electrónica.

\section{Invención del microscopio electrónico de transmisión}

Basado en las ideas de Louis de Broglie, en 1929, Hans Busch indica que es posible enfocar un haz de electrones con campos electromagnéticos (bobinas) de la misma manera en que las ondas de luz se enfocan mediante las lentes de vidrio. Este aporte fue crucial para la construcción del microscopio electrónico. La invención del microscopio electrónico de transmisión (TEM) correspondió a Ernst Ruska y Max Knoll (figura 5), (Brenton, 2004).

De acuerdo con Louis de Broglie, los electrones en movimiento presentan una longitud de onda más pequeña que la luz y, por lo tanto, podrían ofrecer una resolución mejor que el microscopio de luz. Así, en 1931, Ernst Ruska cons-

Figura 5. A) En 1931, Ernst Ruska (derecha) y Max Knoll construyen el prototipo del TEM. B) Enrst August Friedrich Ruska en 1933.
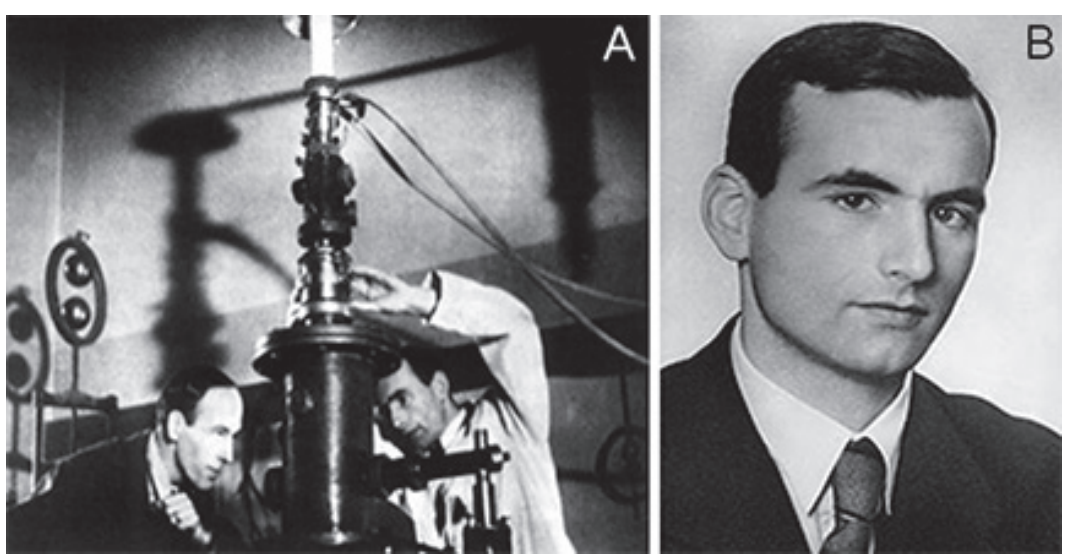

Fuente: www.google.com.mx/searchs
Figura 4. En 1924, el físico francés Louis de Broglie desarrolla la teoría ondutoria del electrón donde se propon onda.

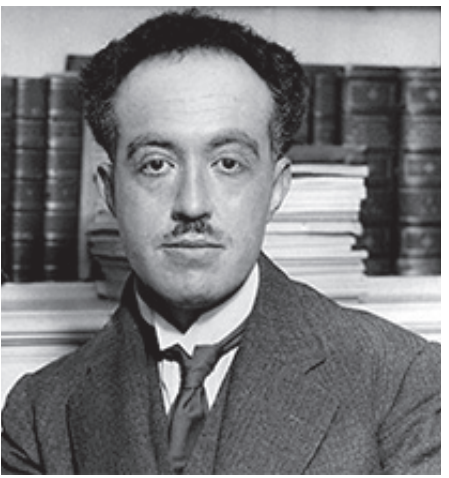

Fuente: www.google.com.mx/search 
truye un microscopio cuya fuente de iluminación era un conjunto de electrones acelerados (el haz electrónico) bajo la tutoría de Max Knoll (figura 5) y, en 1933, la compañía Siemens presenta el prototipo del TEM de Ruska. En 1938, junto con Bodo von Borries (figura 6), Ruska reporta una resolución de $10 \mathrm{~nm}$ la cual rebasa por mucho la resolución del microscopio de luz. Este microscopio contaba con un recipiente tipo "lavabo" como sistema de enfriamiento por agua (Goldstein, 1992). Este diseño permitió generar los primeros microscopios producidos en serie; tenían un voltaje de aceleración de $70 \mathrm{kV}$ y una resolución de 7 nm. Enrst Ruska recibió el Premio Nobel en 1986 por su invención.

En 1940, James Miller de la Radio Corporation of America (RCA) construye un microscopio electrónico bajo la supervisión de Vladimir K. Zworykin e inventaron, además, el cinescopio de la televisión (Nixon, 1998; Toledo, 2005). La compañía inglesa Vickers Metropolitan produjo el prototipo mejorado del TEM llamado EM1, al mismo tiempo, la compañía Phillips presenta en Holanda un microscopio TEM comercial. El primer modelo comercial de la compañía RCA presentó una resolución de $2.5 \mathrm{~nm}$ y se instaló en la Universidad de Stanford en diciembre de 1940, durante la segunda guerra mundial.

En la Universidad de Toronto, Canadá, también se construye un TEM bajo la supervisión de Eli Franklin Burton, A. Prebus y James Miller. Un instrumento similar es construido en Estados Unidos por Cecil E. Hall. Ladislaus L. Marton construyó un TEM en Bruselas, Bélgica, y obtuvo la imagen de una bacteria (Kimoto, 1985). A pesar del avance tecnológico en el campo de la microscopía electrónica de transmisión, no fue sino hasta la década de los años setenta del siglo XX que Hirsh y colaboradores desarrollaron la teoría de formación de imágenes en el TEM (Hirsch et al., 1977) lo que permitió interpretar correctamente las imágenes de TEM en condición de dos haces (figura 7).

Figura 6. A) Bodo von Borries (1905-1956), colaborador de Ruska y Knoll, y fundador de la Federación Internacional de Sociedades de Microscopía Electrónica (IFSEM). B) Fotografía de mayo de 1932 del grupo de trabajo del Dr. Knoll. Ernts Ruska (sentado, de traje) y Bodo von Borries.
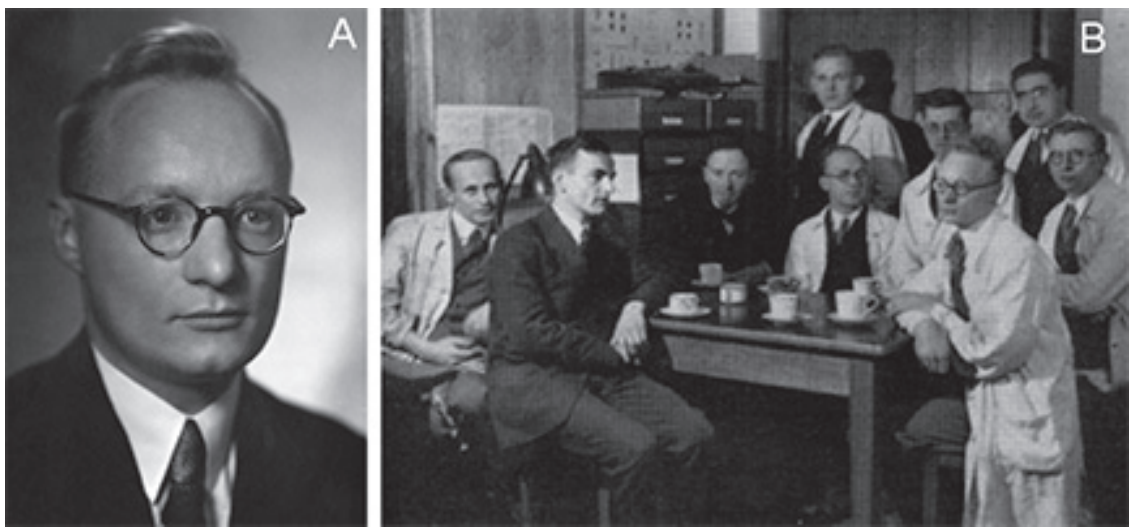

Fuente: www.google.com.mx/searchs 
Mundo Nano | ARTículos DE REVISIóN | www.mundonano.unam.mx

13(25), 79-100, julio-diciembre 2020 | https://doi.org/10.22201/ceiich.24485691e.2020.25.69610

José Reyes Gasga

Figura 7. En la década de los años 70 del siglo XX, Hirsh y colaboradores desarrollan la teoría de formación de imágenes en el TEM
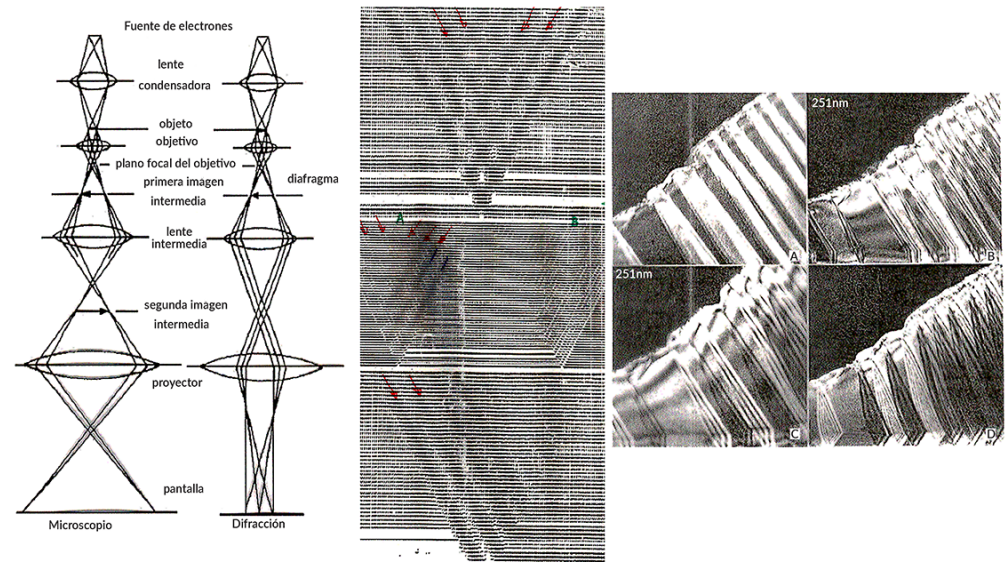

Fuente: Hirsch et al. (1977), Yacamán y Reyes Gasga (1998).

\section{Invención del microscopio electrónico de barrido}

Después de la invención del TEM surgió la propuesta de un microscopio electrónico de barrido (SEM) por el propio Knoll en 1935, pero fue necesario esperar más de 30 años para que el primer SEM saliera al mercado debido a problemas tecnológicos, pues era necesario comprender y solucionar los efectos causados por las aberraciones y lograr un sistema de barrido con suficiente rapidez de respuesta (Goldstein, 1992).

En 1938, Manfred von Ardenne (figura 8) introduce un sistema de barrido dentro del diseño de un TEM, lo cual significó la obtención de un nuevo tipo de microscopio: el microscopio electrónico de barrido-transmisión (STEM, por sus siglas en inglés). Un año después, Boersch logra haces electrónicos con lon-

Figura 8. En 1938, Manfred von Ardenne y el primer microscopio STEM.

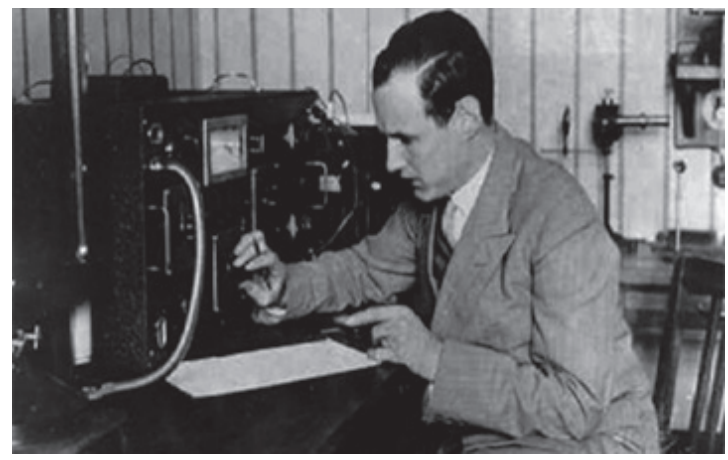

Fuente: www.google.com.mx/searchs 
gitudes de onda del orden de los $2.5 \mathrm{~nm}$, lo que implicó una mejoría considerable en el diseño del SEM (Scott, 1983).

En plena segunda guerra mundial, en 1942, se produce un salto considerable en el desarrollo del SEM con los trabajos de Vladimir K. Zworykin, Charles William Oatley y colaboradores (figura 9) de donde se obtiene un prototipo del detector de electrones secundarios. Para su obtención se aplicó una carga de recolección de $+50 \mathrm{~V}$ a una caja de Faraday y se acopló a un tubo fotomultiplicador (Scott 1983). A pesar de estos aportes, la resolución del microscopio SEM solo llegó a los 50 nm y a 8,000x de aumento. En 1943, se inicia el desarrollo de la espectroscopía por pérdida de energía (EELS).

En 1944, Manfred von Ardenne logra una resolución de $1.5 \mathrm{~nm}$ en el microscopio STEM, pero se presentan importantes problemas de mantenimiento, reparación y, principalmente, de interpretación de las imágenes observadas con el TEM (Hirsch et al., 1977). En 1946, James Hiller obtiene una resolución de 1 nm. En 1947, James Hiller y Edward G. Ramberg desarrollan una lente objetiva con corrección de astigmatismo.

En 1948, bajo la dirección de Charles Williams Oatley, Dennis McMullan (figura 9) desarrolla una fuente estable de alto voltaje para alimentar el filamento de electrones y se introduce en el tubo de rayos catódicos (TRC) como sistema de observación de las imágenes del SEM. Estas nuevas adiciones representaron un importante avance en el desarrollo de la microscopía electrónica (Nixon, 1998).

En 1951, Raimond Castaing inicia el desarrollo de la espectroscopía por rayos X característicos (EDS, por sus siglas en inglés) (Scott, 1983), que al incorporarla al sistema óptico del SEM lo convertía en un instrumento analítico. También, en 1951, James M. Lafferty descubre que los cristales de $\mathrm{LaB}_{6}$ emiten una mayor densidad de electrones que los alambres de W, con un área de emisión correspondiente a un solo átomo, pero requieren de un sistema de vacío

Figura 9. Charles William Oatley y sus estudiantes en una reunión por su 90 aniversario.

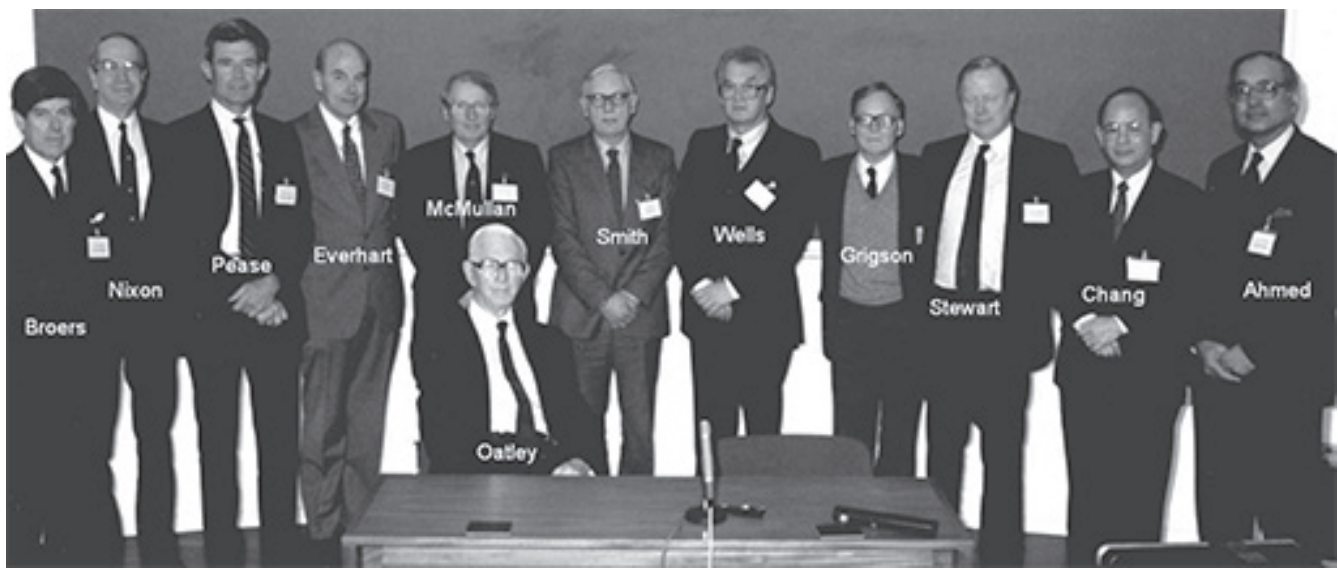

Fuente: www.google.com.mx/searchs 
que produzca un vacío de $10^{-7}$ torrs como mínimo (Kimoto, 1985). A la par, Erwin Wilhem Muller y Kanwar Bahadur inventan el microscopio de campo iónico (FIM) y observan posiciones atómicas por primera vez (Breton, 2004).

En 1956, ocurre otro avance importante en la microscopía electrónica cuando K. C. A. Smith introduce el procesamiento no lineal de las señales, el barrido con doble deflexión del haz, la corrección electromagnética del astigmatismo y un sistema eficaz de centrado de las aperturas. Dando continuidad a los trabajos de Dennis McMullan, Thomas E. Everhart y Richard F. M. Thornley desarrollan, en 1960, un detector basado en el empleo de un dispositivo centellador (Breton, 2004).

\section{Comercialización del microscopio electrónico de barrido}

En los años sesenta, varios fabricantes de microscopios electrónicos trabajaron en el desarrollo de un SEM que pudiera ser comercializado, claro está. La compañía Cambridge Scientific Instruments fabrica el SEM MARK I STEREOSCAN, equipo que sale al mercado en diciembre de 1965 (figura 10), seguido muy de cerca por JEOL, que comercializa su primer SEM en enero de 1966 (Kimoto, 1985). El SEM “Stereoscan 1" tenía 20 kV de voltaje de aceleración y resolución de $30 \mathrm{~nm}$.

Un aporte importante en el desarrollo de la microscopía electrónica estuvo relacionado con el mejoramiento de los filamentos de emisión de electrones. En 1970, los filamentos de Wolframio (W) dominaban el campo. Sin embargo, en 1969, Alec N. Broers presenta el filamento de $\mathrm{LaB}_{6}$, y casi simultáneamente Albert Victor Crewe y J. Wall realizan mejoras en el uso del filamento de Emisión de Campo.

A partir de la década de los setenta del siglo pasado se producen mejoras en las lentes de los microscopios, así como en el desarrollo de diferentes sistemas de

Figura 10. El SEM “Stereoscan 1", fabricado por la compañía Cambridge Instruments en 1964.

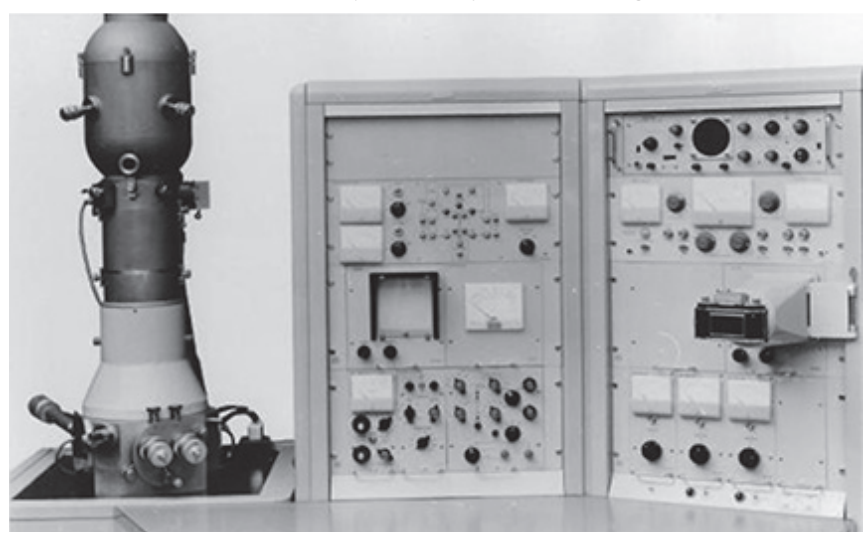

Fuente: www.google.com.mx/searchs 
Figura 11. En 1980, el SEM alcanza una resolución de $2 \mathrm{~nm}$.

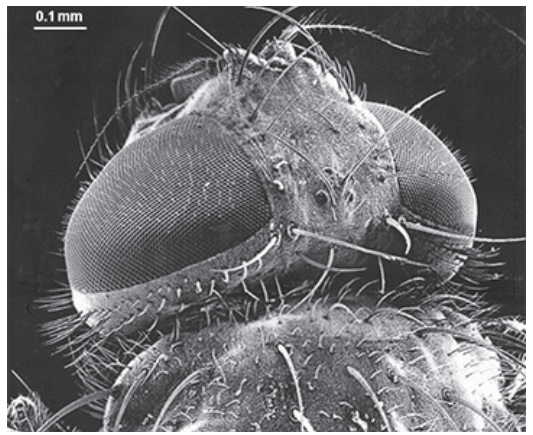

detección. En 1980, el SEM alcanza una resolución de $2 \mathrm{~nm}$ (figura 11). En 1985, la firma Carl Zeiss (que luego pasa a ser la compañía LEO; Stokes, 2012) obtiene un resultado importante al introducir el primer SEM con el barrido controlado de forma digital. Este nuevo componente sería determinante para lograr imágenes ("mapeos") de rayos X mucho más eficaces y para corregir defectos de imagen por acumulación de cargas en la muestra en estudio (Stokes, 2012).

\section{La microscopía electrónica actual}

En 1980, se presentaron mejoras en los sistemas de vacío del TEM con el uso de bombas turbomoleculares y se obtiene la resolución de $0.2 \mathrm{~nm}$. También se desarrollan nuevas técnicas de análisis tales como la difracción electrónica de haz convergente (CBED, por sus siglas en inglés) y mejoras en el funcionamiento del microscopio STEM. El desarrollo de la técnica de difracción de haz convergente da un importante impulso a la cristalografía electrónica. El uso de filamentos de emisión de campo permite a los microscopios SEM alcanzar resoluciones por debajo de $2 \mathrm{~nm}$ y a los microscopios TEM por debajo de $0.1 \mathrm{~nm}$. En 1986, surgen los primeros prototipos de microscopios electrónicos controlados por computadora.

En ese momento se usaron computadoras con un propósito específico y a estos equipos se les llamó de "primera generación", para diferenciarlos de los que surgieron en el año 1992 llamados de "segunda generación" o "G2", en los que una computadora personal toma el control de todo el sistema. En 1990, la compañía Jeol presenta el microscopio JEM-1210 (figuras 12 y 13) operado completamente a través de software computacional (Cadwell, 1996). Las características técnicas de los actuales

Figura 12. Microscopio TEM JEM-1210 de la compañía Jeol operado a través de software computacional.

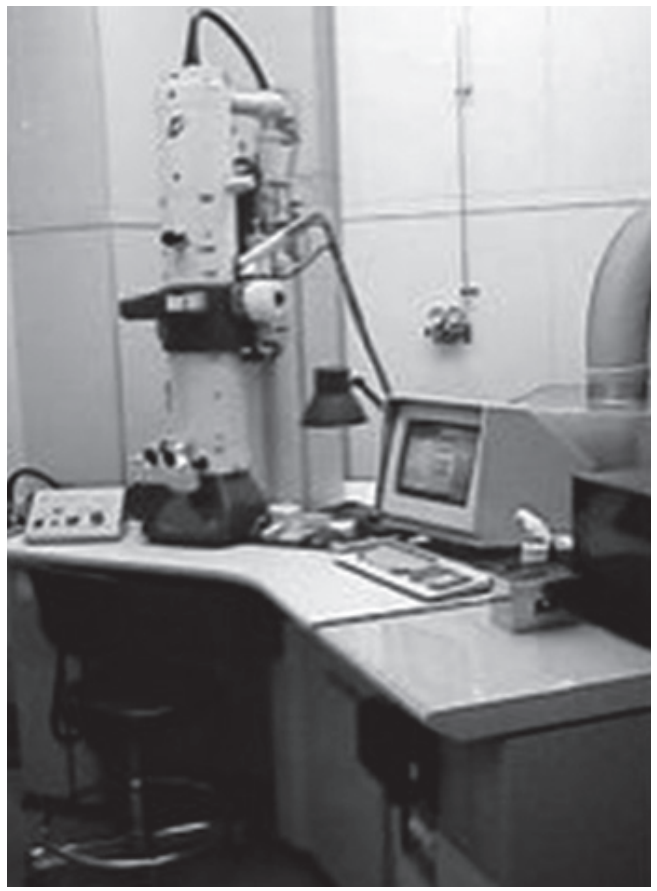

Fuente: www.jp.caeonline.com/jeol-jem-1210. 
Mundo Nano | ARTículos DE REVISIóN | www.mundonano.unam.mx

13(25), 79-100, julio-diciembre 2020 | https://doi.org/10.22201/ceiich.24485691e.2020.25.69610

José Reyes Gasga

Figura 13. Los microscopios electrónicos actuales son operados a través del software de una computadora.
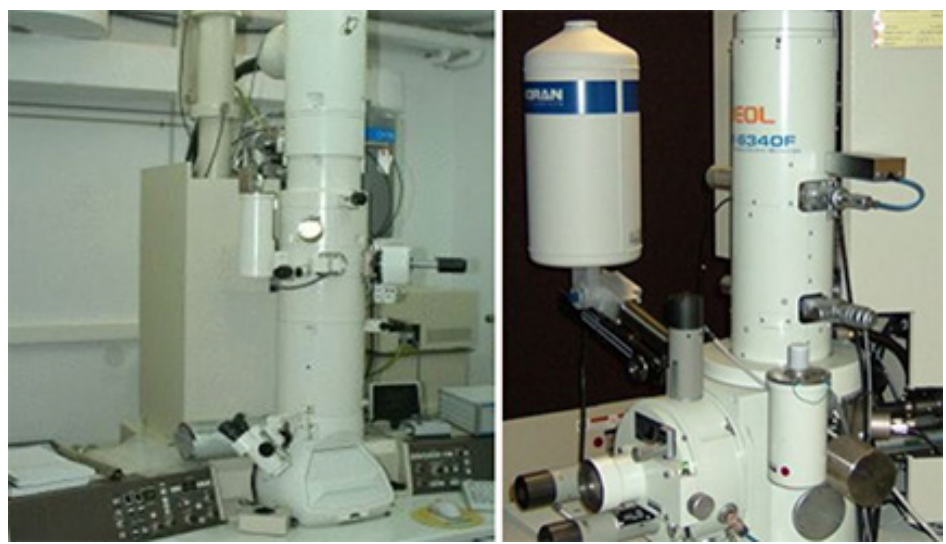

Fuente: www.es.m.wikipedia.org/wiki/archivoJEOL.JSM-6340F

microscopios permiten estudiar in situ las transformaciones estructurales de los materiales (figura 14).

De manera paralela, en 1981, Heinrich Roher y Gerd Binnig, investigadores de la IBM de Zurich, Alemania, inventan el microscopio de barrido de tunelaje (STM), el cual revela la topografía de la superficie de la muestra a nivel atómico (Scott, 1983). En 1986, Binnig, Quate y Gerber inventan el microscopio de fuerza atómica (AFM). Ellos junto con Ernst Ruska reciben el Premio Nobel el 10 de diciembre de 1986 (figura 15). De

Figura 14. Las características técnicas de los actuales microscopios permiten estudiar in situ las transformaciones estructurales de los materiales.

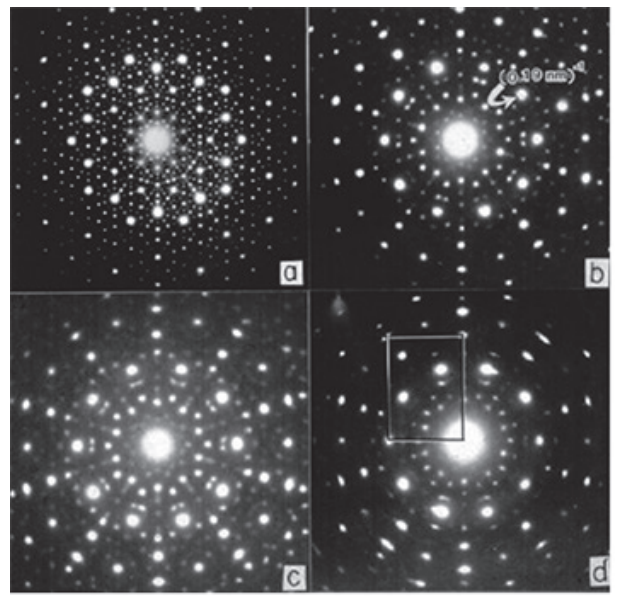

Fuente: Yacamán y Reyes-Gasga (1998). 
Figura 15. El 10 de diciembre de 1986, Ernst Ruska recibe el Premio Nobel por la invención del microscopio electrónico.
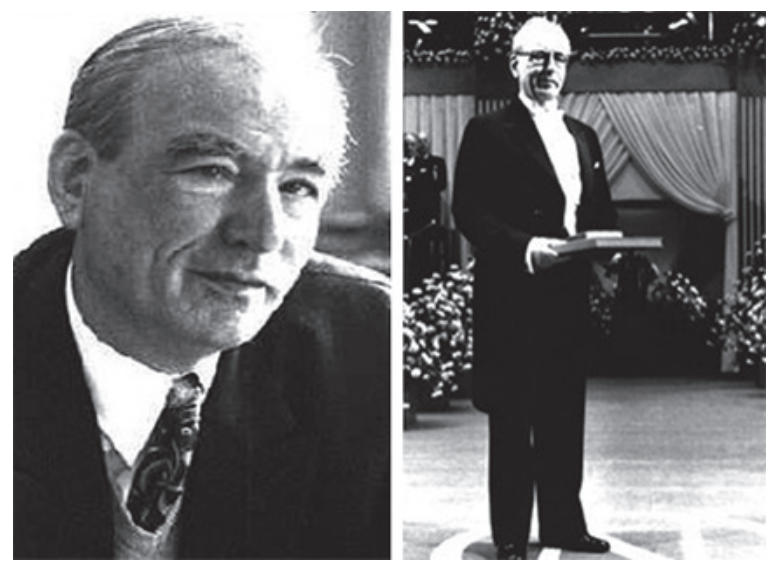

Fuente: www.google.com.mx/searchs

acuerdo con la Sociedad Nobel, el TEM es "uno de los inventos más importantes del siglo XX".

A finales de la década de los años ochenta y principios de los noventa del siglo XX, tuvo lugar otro avance importante en la microscopía electrónica de barrido. Hasta ese momento, se había considerado como inevitable que la muestra observada en un microscopio electrónico debía estar al alto vacío, condición que imponían tanto el camino libre de los electrones y la duración del filamento. En este periodo la compañía Philips (luego llamada FEI) desarrolla la opción de diferenciar el vacío del cañón y la columna del microscopio con el vacío de la cámara donde se coloca la muestra. Estos microscopios SEM se conocen como ambientales (Stokes, 2012).

En 1995, Ondrej Krivanek desarrolla el filtro de energía (los prismas electrónicos) los cuales son actualmente usados como monocromadores en el cañón electrónico y como seleccionadores de energía en la espectroscopía por pérdida de energía (EELS). En los últimos años se ha visto el surgimiento de la microscopía remota controlada vía Internet, lo cual facilita el estudio de muestras a distancia (Breton, 1997). También se ha trabajado con éxito en la obtención de equipos "inteligentes" capaces de fijar las condiciones de trabajo con un alto grado de independencia, a partir de algunos datos elementales sobre la muestra (Caldwell, 1996).

En la actualidad, la evolución de la microscopía electrónica no se ha detenido. Aunque han surgido otros microscopios como los de fuerza atómica con resoluciones en el rango atómico, el SEM y el TEM conservan aún un importante sector en los requerimientos de una gran cantidad de ramas del conocimiento humano. En el campo de la microscopía TEM se ha avanzado decididamente en la obtención de la microscopía de aberración corregida 
(Lentzen, 2006). En 2010, se desarrollaron los detectores para la dispersión a alto ángulo (contraste Z) por Stephen J. Pennycook y colaboradores y el sistema del STEM alcanzó la resolución de 50 picómetros. No cabe duda de que en el futuro la microscopía electrónica continuará prestando un invaluable servicio a la comunidad científica en los campos de la física, matemáticas, química, biología, arqueología, la medicina, la botánica, la ciencia de materiales y las ciencias forenses, por mencionar algunas.

Datos adicionales sobre la evolución de las técnicas de microscopía para el estudio de materiales y de nanomateriales se pueden consultar en Rivera et al. (2019).

\section{La microscopía electrónica en México}

El desarrollo de la microscopía electrónica en México se inicia con la llegada del microscopio de luz en nuestro país, lo cual sucede en 1690, es decir, un siglo después de su invención, cuando Carlos Sigüenza y Góngora trae a México un microscopio tipo Hooke para estudiar un problema de plaga en el maíz (Martínez-Mena, 1988). Sin embargo, no existe reporte alguno sobre este tema salvo una carta familiar donde se hace el comentario. La primera investigación formal realizada y reportada con un microscopio de luz data de 1869 en la revista La Naturaleza, volumen I, página 26, con el título El microscopio y la fotografía aplicados a las ciencias naturales, y firmado por José Joaquín Arriaga. A principios del siglo XX, Isaac Ochotorena publica 3 trabajos en el área de la histología con el microscopio de luz como herramienta básica. El primer curso de enseñanza formal en microscopía de luz data de los años veinte del siglo XX (Martínez-Mena, 1988). Fue en el área de la botánica y fue dictado por Enrique Beltrán en la Facultad de Altos Estudios (que después sería la Facultad de Filosofía y Letras de la UNAM). Este curso quedó registrado como "Curso libre de microscopía botánica". En 1941, Enrique Beltrán publica el artículo "El microscopio electrónico y sus posibilidades" en la revista mexicana Ciencia.

\section{La microscopía electrónica de barrido en México}

La microscopía electrónica en México se inició en 1946 cuando se fundó el Laboratorio de Microscopía Electrónica en la Escuela Nacional de Ciencias Biológicas del IPN (Martínez-Mena, 1988; Yacamán y Krakow, 1998). El primer microscopio electrónico en México fue un microscopio SEM de la marca RCA modelo I-MC-1 con $30 \mathrm{kV}$ de aceleración, resolución de $5 \mathrm{~nm}$ y con amplificaciones de hasta 5,000x. Con este equipo se estudiaron muestras biológicas y puede decirse que los primeros microscopistas electrónicos fueron Antonio González Ochoa y Gerardo Varela, quienes estudiaron agentes microbiológicos. En 1949, Nicolás Aguilera Herrera (figura 16) presenta la primera tesis de licenciatura realizada en México usando la microscopía electrónica. El maes- 
tro Aguilera obtuvo la licenciatura de químico bacteriólogo parasitólogo con la tesis Estudio de arcillas y suelos de México usando microscopía electrónica. En este trabajo, el maestro Aguilera y el físico Efrén Fierro utilizan el microscopio SEM para el estudio de arcillas marcando así el inicio de la investigación en microscopía electrónica aplicada a los suelos de México y Latinoamérica. Por esta razón, el maestro Aguilera es considerado como el pionero en la aplicación de la microscopía electrónica al estudio de las arcillas de suelos en México. En 1950, el doctor Jorge González Ramírez, del Instituto de Estudios Médicos y Biológicos del IPN, realiza estudios de tejidos usando un SEM, y es el primer mexicano en llevar a cabo estudios de microscopía electrónica de alto nivel en el área biológica (Martínez-Mena, 1988).

Un segundo microscopio SEM de la marca RCA modelo Universal 2A con 50 kV de aceleración y 2.5 nm de resolución llegó a México en 1951 a la Escuela Nacional de Agricultura para realizar el estudio de la estructura de los suelos mexicanos. En la década de los años sesenta del siglo pasado, la UNAM adquiere varios microscopios electrónicos SEM para las Facultades de Medicina y la de Ciencias y para el Instituto de Física. Se puede decir que los cimientos de la microscopía electrónica en México se establecieron entre 1960 y 1970, principalmente en el área de las ciencias biológicas. Varios hospitales y laboratorios del sector público también adquirieron microscopios SEM (Yacamán y Krakow, 1998).

Figura 16. Nicolás Aguilera Herrera.

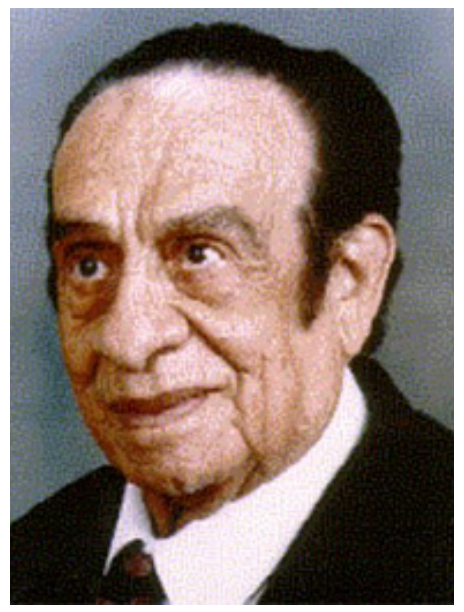

Nota: Nació en Yuriria, Guanajuato, el 21 de octubre de 1920. Realizó estudios en la Escuela Nacional de Ciencias Biológicas del IPN. Inició su actividad docente en 1949 en la Escuela Nacional de Agricultura, dictando un curso de microscopía. En el Colegio de Postgraduados, realizó investigación sobre los suelos derivados de las cenizas volcánicas de la parte central de México. En 1965, en el Departamento de Biología de la Facultad de Ciencias de la UNAM, produce un número considerable de trabajos en el área de microscopía electrónica de barrido e imparte cursos y conferencias sobre este tema. El Colegio de Postgraduados le otorgó el Doctorado Honoris Causa, unos días antes de su fallecimiento, acaecido el 7 de septiembre de 1996.

Fuente: www.fciencias.unam.mx/vida-en-ciencias/distinciones. 
Mundo Nano | ARTículos DE REVISIóN | www.mundonano.unam.mx

13(25), 79-100, julio-diciembre 2020 | https:// doi.org/10.22201/ceiich.24485691e.2020.25.69610

José Reyes Gasga

Figura 17. Adolfo Martínez Palomo.

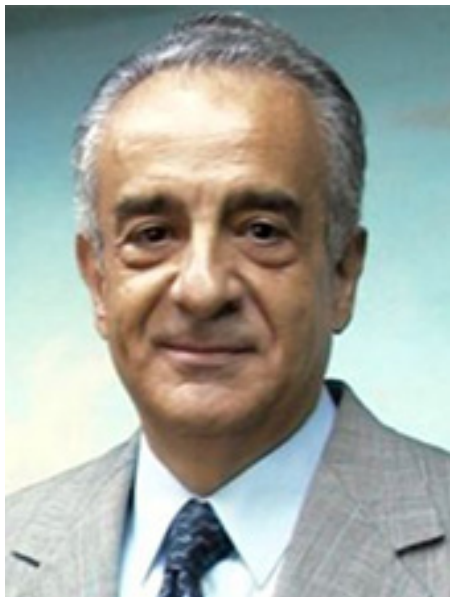

Nota: Nació en la Ciudad de México en 1941. Es médico cirujano por la Facultad de Medicina de la UNAM. Realizó el doctorado en Ciencias Médicas de la Facultad de Medicina de la UNAM en 1971 y fue jefe del Laboratorio de Microscopía Electrónica del Instituto Nacional de Cardiología de 1968 a 1972. Ha sido miembro de Juntas de Gobierno de la UNAM y de El Colegio de México. Ha participado en la Comisión de Investigación en Salud de la Universidad de Harvard, el Foro Global para la Investigación en Salud (Ginebra), y los comités asesores de investigación en salud de la Organización Mundial de la Salud (Ginebra) y de la Organización Panamericana de la Salud (Washington, EUA). Fue miembro de comités científicos de la Fundación Rockefeller, del Instituto Internacional de Vacunas (Seúl) y del Centro de Prevención y Control de Enfermedades (CDCP, Atlanta), así como presidente del Comité Internacional de Bioética de la UNESCO (París). Es miembro de El Colegio Nacional e investigador emérito del Sistema Nacional de Investigadores y profesor emérito del Cinvestav del IPN, institución de la que fue director general (1995-2002), fundador y jefe del Departamento de Patología Experimental.

Fuente: www.google.com.mx/search

Uno de los principales impulsores de la microscopía electrónica en México en el área de las ciencias biológicas fue el doctor Adolfo Martínez Palomo (figura 17), quien hizo su tesis profesional con el doctor Issac Costero, creador de la Escuela de Patología de la UNAM (Martínez-Mena 1988). En 1966, Martínez Palomo estudió microscopía electrónica en Francia y en cuya estancia fue pionero en ver las uniones en los contactos entre células tumorales con el microscopio electrónico. Durante varios años de investigación en el Instituto Nacional de Cardiología, estudió el sistema de conducción del corazón con el microscopio electrónico.

\section{Microscopios electrónicos de transmisión en México}

El primer microscopio dedicado a la investigación en el área de ciencia de materiales fue instalado en el Centro de Investigación y de Estudios Avanzados del IPN bajo la dirección de Juan Luís del Valle (Yacamán y Kravov, 1998). El primer microscopio electrónico de transmisión en la UNAM dedicado al estudio de materiales llegó como donación al Instituto de Física. Este 
fue un microscopio electrónico Phillips 100 horizontal modelo 1952. Entre los pioneros de la microscopía electrónica en los estudios de materiales se encuentra el doctor Gabriel Torres Villaseñor (figura 18), quien fungió como coordinador de dicho microscopio.

A mediados de los años setenta del siglo XX, se instala en el Instituto de Física de la UNAM un microscopio electrónico TEM de la marca Jeol modelo $200 C X$ con resolución de $0.3 \mathrm{~nm}$ para el grupo encabezado por el doctor $\mathrm{Mi}$ guel José Yacamán. Casi al mismo tiempo, se instala otro microscopio similar en el Instituto de Investigaciones en Materiales de la UNAM, para el grupo dirigido por el doctor Gabriel Torres Villaseñor. Es precisamente con estos microscopios que la microscopía electrónica TEM en México alcanza niveles de calidad internacional en el área de las ciencias físicas y de materiales. El doctor Yacamán (figura 19) y sus estudiantes, los doctores David Romeu Casajuana, Alfredo Gomez Rodriguez y Pablo Schabes, realizan una serie de trabajos de alto nivel en la interpretación del contraste observado en los microscopios TEM (Yacamán y Reyes-Gasga, 1998). En 1984, el Instituto de Física de la UNAM transfirió este microscopio electrónico Jeol 200CX al Departamento de Nanoestructuras del Centro de Nanociencias y Nanotecnología de la UNAM en Ensenada, Baja California Norte, que en aquel tiempo formaba parte del instituto (figura 20).

Figura 18. Gabriel Torres Villaseñor.

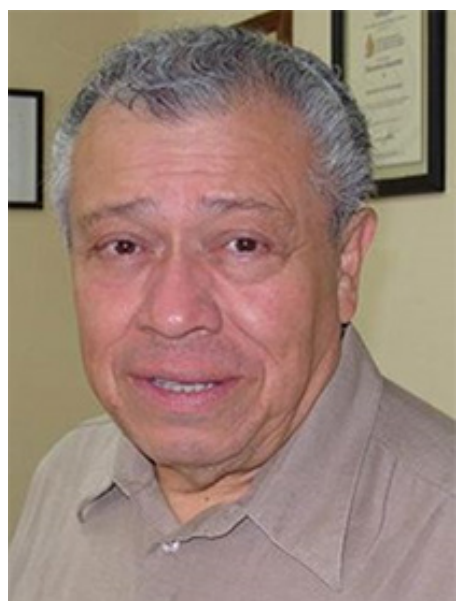

Nota: Nació en la Ciudad de México en 1944. Obtuvo la licenciatura y la maestría en Física en la Facultad de Ciencias de la UNAM y el doctorado en Ciencias de Materiales en Case Western Reserve University, en Cleveland, Ohio, EUA. Fue jefe del Departamento de Metalurgia y Cerámicos del Instituto de Investigaciones en Materiales de la UNAM y miembro del Consejo Universitario, del Consejo Consultivo de Ciencias de la Presidencia de la República y del Editorial Advisory Board of Materials Science Foundations de EUA. Recibió el Premio Nacional de Ciencias y Artes y el premio Manuel Noriega en 1984, otorgado por la Organización de Estados Americanos. Actualmente, es Investigador Emérito de la UNAM en el Instituto de Investigación en Materiales de la UNAM.

Fuente: Archivo personal del autor. 
Mundo Nano | ARTículos DE REVISIóN | www.mundonano.unam.mx

13(25), 79-100, julio-diciembre 2020 | https://doi.org/10.22201/ceiich.24485691e.2020.25.69610

José Reyes Gasga

Figura 19. Miguel José Yacamán.

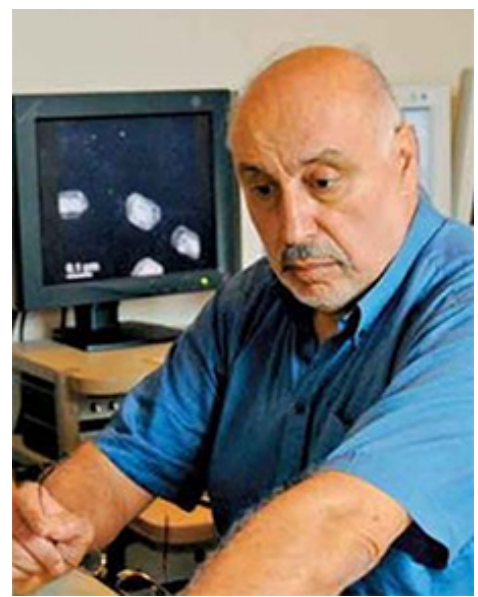

Nota: Nació en la ciudad de Córdoba, Veracruz, en 1946. Desarrolló su tesis de licenciatura en física con el Dr. Alonso Fernández y obtuvo el doctorado en Física en la Facultad de Ciencias de la UNAM en 1973, realizando su tesis doctoral con el Dr. Eduardo Muñoz Picone, en colaboración con Alan Basset, primero en Cambridge y luego en Warwick. Sus campos de especialidad son la ciencia de materiales, caracterización de materiales por microscopía, difracción de electrones y materiales nanoestructurados. Fue director del Instituto de Física de la UNAM; director adjunto de Investigación Científica del Conacyt; secretario ejecutivo del Sistema Nacional de Investigadores; director general del Instituto Nacional de Investigaciones Nucleares. Profesor de la Universidad de Texas en Austin y en San Antonio. Perteneció al Consejo Consultivo de Ciencias de la Presidencia. Recibió la Beca Guggenheim; el Premio de la Academia de la Investigación Científica; la presea del Estado de México "Antonio Alzate" en Ciencias Exactas; el Premio Nacional de Ciencias y Artes y The Mehl Award and Distinguish Lecturer of The Metals Society TMS de Estados Unidos.

Fuente: www.google.com.mx/search

Figura 20. Microscopio electrónico Jeol 200CX en el Departamento de Nanoestructuras del Centro de Nanociencias y Nanotecnología de la UNAM, en Ensenada, Baja California Norte.

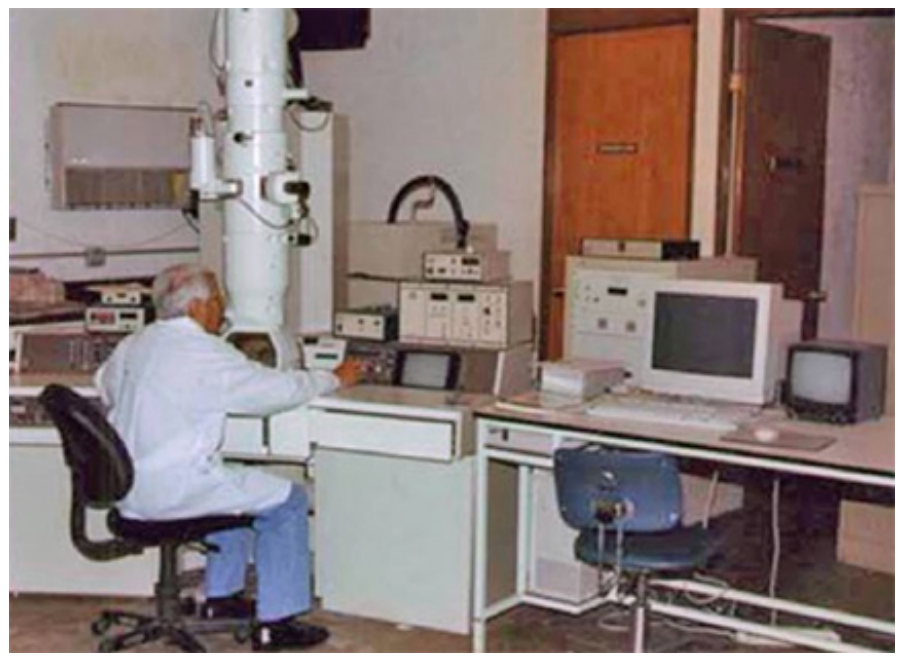

Fuente: Archivo personal del autor. 
Figura 21. Microscopio electrónico Jeol 100CX, instalado en el Instituto de Física de la UNAM en 1982.
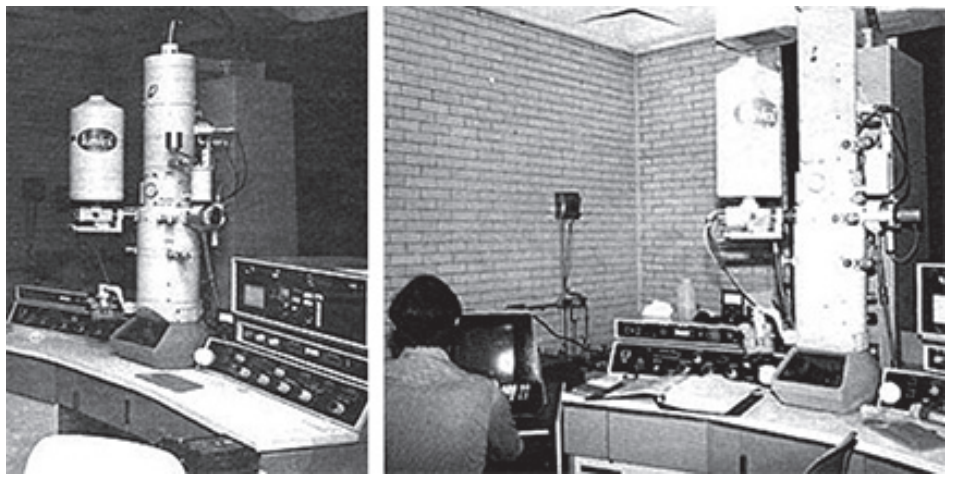

Fuente: Archivo personal del autor.

En 1982 se instala en el Instituto de Física de la UNAM el microscopio electrónico de transmisión y barrido (STEM) Jeol 100CX (figura 21) de 100 $\mathrm{kV}$ de voltaje de aceleración, que cuenta con un detector de rayos $\mathrm{X}$ característicos (EDS) y con resolución de $0.25 \mathrm{~nm}$, dedicado a la ciencia de materiales. En 1988 se instala nuevamente en el Instituto de Física de la UNAM el microscopio electrónico de alta resolución Jeol 4000EX (figura 22) con voltaje de aceleración de $400 \mathrm{kV}$ y resolución de $0.17 \mathrm{~nm}$, primero de su tipo que se instala en Latinoamérica.

A inicios de los años noventa del siglo XX, la microscopía TEM en la ciencia de los materiales y en ciencias biológicas alcanza los niveles de alta

Figura 22. Microscopio electrónico de alta resolución Jeol 4000EX instalado en el Instituto de Física de la UNAM en 1988.

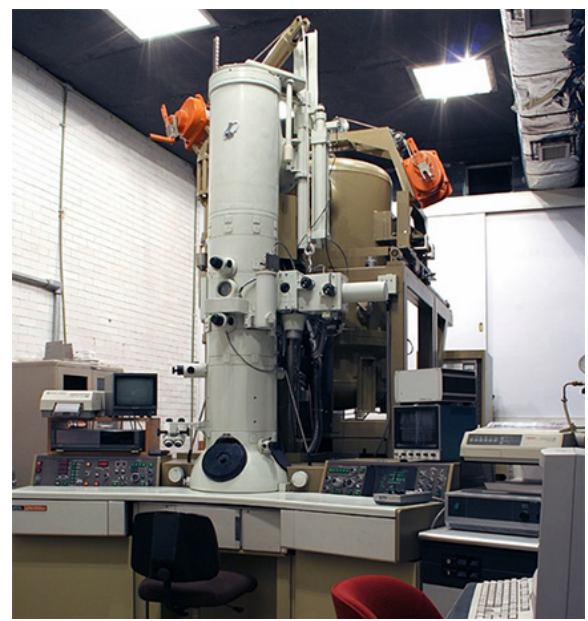

Fuente: Archivo personal del autor. 
especialidad y calidad en México con un número considerable de artículos de investigación en revistas de circulación internacional y de formación de recursos humanos.

La Asociación Mexicana de Microscopía fue fundada el 9 de noviembre de 1991. Esta fue establecida por los doctores Miguel José Yacamán, Jorge Larriva, Alfonso Cárabez Trejo, David Ríos Jara, Pablo Schabes Retchkiman, Victor Tsutsumi y Leonel Cota Araiza. Durante el evento de fundación de la Asociación, el doctor Ruy Pérez Tamayo, de la División de Medicina Experimental de la Facultad de Medicina de la UNAM, vaticinó que la Asociación Mexicana de Microscopía tendría una vida cada vez más activa a medida que se fuese fortaleciendo. El doctor Ciro Falconi, responsable de la Unidad de Microscopía del CINVESTAV, expresó que las continuas innovaciones en la microscopía obligan a los expertos a una permanente actualización. El doctor Gerardo Vázquez Nin del Laboratorio de Microscopía Electrónica del Departamento de Biología de la Facultad de Ciencias de la UNAM indicó la necesidad de contar con personal capacitado para manejar los microscopios electrónicos, por lo que la Facultad de Ciencias estableció una especialización en microscopía electrónica en el área biológica a nivel posgrado. Además, comprometió a la Asociación Mexicana de Microscopía a establecer una serie de cursos de microscopía electrónica tanto en el área de las ciencias biológicas como en el área de las ciencias físicas. El doctor René Druker, del Instituto de Fisiología Celular de la UNAM, consideró que la creación de la Asociación fue un paso muy acertado al existir excelentes microscopistas en México.

En 1992, la Asociación Mexicana de Microscopía en conjunto con las Sociedades de Microscopía Electrónica de Venezuela, Brasil, Argentina, Estados Unidos y Cuba establecen, durante el Primer Congreso Atlántico de Microscopía Electrónica, celebrado en Mérida, Venezuela, del 25 al 29 de mayo, el Comité Interamericano de Sociedades de Microscopía Electrónica (CIASEM), con el objetivo de incrementar y difundir la ciencia y la enseñanza de la microscopía electrónica en el continente americano, y pasa a formar parte de la Confederación Internacional de Sociedades en Microscopía Electrónica (IFSEM) fundada en 1951 por Bodo von Borries, colaborador de Ruska y Knoll. El doctor Miguel José Yacamán fue el primer presidente del CIASEM, y cabe mencionar que México ha sido siempre pieza clave en la organización y funcionamiento del CIASEM.

En la década de los años noventa, México cuenta con varios microscopios electrónicos en varias universidades del país. La Asociación tuvo un papel importante en la elección de México como sede del II Congreso Iberoamericano de Microscopía Electrónica, realizado en 1993, y, en 1994, la IFSEM en su Asamblea General celebrada en París, Francia, confiere a México la organización y celebración del 14 Congreso Internacional de Microscopía Electrónica, celebrado en Cancún, Quintana Roo, en septiembre de 1998, con la participación de más de 1500 microscopistas de todo el mundo (Calderón-Benavides et al., 1998). 
En la actualidad hay más de 100 microscopios electrónicos operando en México, tanto SEM como TEM, siendo uno de los países latinoamericanos con mayor número de microscopios. Los laboratorios de microscopía electrónica en México se encuentran instalados principalmente en la UNAM, el IPN y en el sector salud, pero en casi todos los estados del país se tiene al menos un laboratorio de microscopía electrónica, principalmente en las instalaciones de las universidades estatales y en los tecnológicos. En 1998 la revista Microscopy Research and Technique dedicó un número especial al trabajo de microscopía electrónica realizado en México. Varios de los actuales investigadores en microscopía electrónica, tanto en el área de las ciencias biológicas como en el área de ciencia de materiales, publicaron parte de su trabajo en dicho volumen.

Hoy en día, también se imparte un número considerable de cursos en microscopía electrónica en varias dependencias de la UNAM, del IPN y en varias universidades del país. Por ejemplo, en el Laboratorio de Microscopía Electrónica de la Facultad de Ciencias de la UNAM, se imparte la especialización en microscopía electrónica aplicada a las ciencias biológicas, como parte del posgrado de la UNAM.

Con respecto a los equipos modernos, tanto el IPN (en el Laboratorio Avanzado de Nanoscopía Electrónica del CINVESTAV y en el Centro de Nanociencias y Micro y Nanotecnologías, del IPN), la UNAM (en el Instituto de Investigaciones en Materiales) y el Instituto Mexicano del Petróleo cuentan actualmente con el microscopio electrónico Jeol ARM200F, con corrector de aberración esférica, el cual permite obtener imágenes en el modo de barrido por transmisión (HAADF-STEM) con una resolución de 78 picómetros $(0.078 \mathrm{~nm})$. La resolución con que se cuenta en los microscopios TEM de última generación es de 50 picómetros, es decir, dentro del mundo atómico, y la física cuántica tiene la palabra.

\section{Comentarios finales}

La actividad actual en los laboratorios de microscopía electrónica a nivel mundial se ha modificado considerablemente. En un principio, las actividades se enfocaron completamente en la observación de diferentes materiales con el microscopio electrónico y en la interpretación y análisis de las imágenes. Ahora, las actividades se pueden dividir en aquellas que se interesan por el desarrollo de la ciencia de la microscopía electrónica per se y las que se interesan solo por la técnica, pero estas actividades no son excluyentes.

El objetivo primordial de la microscopía electrónica como ciencia es aumentar la resolución y calidad, e interpretar las imágenes que se obtienen con el SEM y TEM. Para mejorar la resolución y la calidad de las imágenes se requiere el entendimiento completo de la óptica electrónica; la interpretación de imágenes requiere el conocimiento de la interacción del haz electrónico con los materiales en estudio. Por otro lado, dar servicio a los usuarios de la micros- 
copía electrónica implica usarla solo como una técnica que aporta información sobre la morfología, estructura y composición química de las muestras. Tanto la ciencia como la técnica son actividades muy importantes y no son excluyentes, pero requieren de diferente tiempo de uso de los equipos. Un laboratorio de microscopía electrónica moderno requiere de científicos dedicados a la ciencia de la microscopía electrónica y técnicos dedicados a dar los servicios, pero ambos serán responsables de las actividades de enseñanza de la microscopía electrónica.

En cuestión de equipo, un laboratorio de microscopía moderno estará conformado por al menos un par de microscopios SEM, de mediana y de última generación, y al menos un microscopio TEM de última generación. De igual modo, debe contar con una serie de equipos de preparación y manejo de muestras. La generación de recursos para el mantenimiento recaerá casi en su totalidad en los microscopios SEM y en el equipo de preparación de muestras. La realización de la ciencia recaerá casi completamente en el equipo TEM.

El microscopio electrónico se inventó y perfeccionó en los años treinta del siglo XX, pero en México, la microscopía electrónica se desarrolló en los setenta, prácticamente 50 años después. Actualmente, la microscopía electrónica realizada en México tiene reconocimiento internacional, pero está muy lejos de encontrarse en la frontera de la investigación en esta área.

Realizar investigación en el campo de la microscopía electrónica en México requiere de un apoyo económico considerable, tanto por el costo de estos equipos (se cotizan en más de un millón de dólares), como por el costo de mantenimiento y consumibles, cifra que puede alcanzar los 100 mil dólares anuales.

Así, en la mayoría de los laboratorios de microscopía los directivos han optado por los servicios, por lo cual la mayoría de las actividades de microscopía electrónica se centran en la generación de fondos para poder cubrir el mantenimiento y el costo de los materiales de consumo. Sin embargo, en México, el único microscopio que puede generar los recursos económicos requeridos es el SEM, al ser un equipo que permite obtener en un tiempo relativamente corto las imágenes reconocibles del objeto de estudio, así como su composición química. Si se cuenta con los detectores adecuados, es posible obtener, además, las propiedades ópticas y electrónicas de las muestras.

Otra limitante es que son los técnicos del laboratorio quienes manejan los microscopios electrónicos mientras que los estudiantes y usuarios son meros espectadores. Esta situación ha repercutido en el poco interés del estudiante por el desarrollo de temas relacionados con la microscopía electrónica, reduciendo así la formación de recursos humanos. Como resultado, se genera también un déficit en el personal requerido para el funcionamiento de los laboratorios de microscopía electrónica. Las personas que tienen la necesidad de usar este equipo, la mayoría de las veces solo buscan obtener las imágenes, sin importar el profundizar en los resultados. Una imagen bonita es más que suficiente. 
Hacer ciencia de frontera en el campo de la microscopía electrónica requiere, por consiguiente, una cantidad considerable de recursos económicos anuales y la formación de recursos humanos de las últimas generaciones. Definitivamente, en nuestro país esto implica la colaboración interinstitucional y gubernamental, si es que se quiere que la ciencia en el campo de la microscopía electrónica sea una opción para nuestros futuros científicos

\section{Referencias}

Breton, B. C., Chand, G., Caldwell, N. H. M., Holburn, D. M. (1997). Remote microscopy in the real world. Microscopy and Analysis (Eur. Edn.), septiembre: 9-21.

Breton, B. C. (2004). The early history and development of the scanning electron microscope. Engineering Department, Cambridge University. www-g.eng.cam.ac. $\mathrm{uk} / 125 /$ achievements/oatley/history.html

Calderón Benavides, H. A., José Yacamán M. (1998). Proceedings of the 14th-ICEM, México, Institute of Physics Publising, Bristol. Vols. I, II y III. ISBN-10: 0750305681. ISBN-13: 9780750305686.

Caldwell, N. H. M., Breton, B. C., Holburn, D. M. (1998). Making SEMs smarter. Microscopy and Analysis, noviembre: 9-11. Wiley Analytical Science.

Goldstein, J. I. (1992). Scanning electron microscopy and X-ray microanalysis. Nueva York: Plenum Press. ISBN 978-1-4613-0491-3.

Hirsch, P., Howie, A., Nicholson, R. B., Pashley, D. W., Whelan, M. J. (1977). Electron microscopy of thin crystals. Nueva York: Krieger Publishing Company, NY. ISBN13: 978-0882753768. ISBN-10: 0882753762.

Kimoto, S. (1985). The scanning microscope as a system. JEOL News, Jeol Ltd.

Lentzen, M. (2006). Progress in aberration-corrected high resolution TEM using hardware aberration correction. Microscopy Microanalysis, 12: 191-205. https:// doi.org/10.1017/S1431927606060326

Martínez-Mena, A. (1988). Algunos aspectos de la historia de la microscopía en México. Revista Ciencias: Revista de Cultura Científica. Facultad de Ciencias, UNAM. 25-28. ISSN: 0187-6376.

Nixon, W. (1998). History and early developments of the scanning electron microscopy. Proceedings of the 14th-ICEM. Institute of Physics Publising, Mexico.

Rivera, M., Arenas-Alatorre, J. (2019). De los micrómetros a los picómetros: evolución de las técnicas de microscopía para el estudio de nanomateriales. Mundo Nano. Revista Interdisciplinaria en Nanociencias y Nanotecnología, 12 (23): 1e25e. https://doi.org/10.22201/ceiich.24485691e.2019.23.67334

Scott, V. D., Love, G. (1983). Quantitative electron-probe microanalysis. Ellis Horwood Ltd. ISBN-10:0853125147. ISBN-13: 978-0853125143.

Stokes, D. J. (2012). Environmental scanning electron microscopy for biology and polymer science. Microscopy and Analysis, 67-71. www.microscopy-analysis. com

Toledo, C. A., Varela, R. (2005). 40 años del microscopio electrónico de barrido. Proc. V. Congreso de Microscopía Electrónica, Habana, Cuba. www.sld.cu/galerias/pdf 
Mundo Nano | ARTículos de REVISı́ón | www.mundonano.unam.mx

13(25), 79-100, julio-diciembre 2020 | https:// doi.org/10.22201/ceiich.24485691e.2020.25.69610

José Reyes Gasga

Yacamán, M. J., Krakow, W. (1998). Introduction to microscopy in Mexico. Microscopy Research and Technique, 40(1): 1.

Yacamán, M. J., Reyes-Gasga, J. (1998). Microscopía electrónica: una visión del microcosmos. México: Fondo de Cultura Económica. ISBN-10: 9681646215, ISBN13: 978-9681646219. 